\title{
Inhibition of LPS-Induced PGE2 Production by Arylsulfonamide Derivatives via the Selective Inhibition of mPGES-1 Enzyme ${ }^{\dagger}$
}

\author{
Misong Kim ${ }^{1, *}$, Changyoung Jang ${ }^{1}$, Yunchan Nam ${ }^{1}$, Kyung-Tae Lee ${ }^{2}$ and Jaeyeol Lee 1,3 \\ 1 Research Institute for Basic Sciences and Department of Chemistry, College of Sciences, \\ Kyung Hee University, Seoul 02447, Korea \\ 2 Department of Life and Nanopharmaceutical Science, Kyung Hee University, Seoul 02447, Korea \\ 3 KHU-KIST Department of Converging Science and Technology, Kyung Hee University, Seoul 02447, Korea \\ * Correspondence: miisong@khu.ac.kr \\ + Presented at the 2nd Molecules Medicinal Chemistry Symposium (MMCS): Facing Novel Challenges in \\ Drug Discovery, Barcelona, Spain, 15-17 May 2019. \\ Published: 7 August 2019
}

\begin{abstract}
Microsomal prostaglandin E synthase-1 (mPGES-1) is responsible for the massive prostaglandin $\mathrm{E}_{2}\left(\mathrm{PGE}_{2}\right)$ formation during inflammation. Increasing evidence reveals mPGES-1 inhibitors as a safe alternative to nonsteroidal anti-inflammatory drugs. Recently, we reported that a novel series of phenylsulfonyl hydrazide derivatives could reduce LPS-induced PGE2 levels in RAW 264.7 macrophage cells via an inhibition of the mPGES-1 enzyme. However, a few of the phenylsulfonyl hydrazide derivatives showed poor metabolic stability in liver microsomes. In order to identify new mPGES-1 inhibitors with improved metabolic stability, therefore, a series of arylsulfonamide derivatives has been synthesized and biologically evaluated against PGE2 production and the mPGES-1 enzyme. Among them, MPO-0186 inhibits the production of PGE2 $\left(\mathrm{IC}_{50}=0.20 \mu \mathrm{M}\right)$ in A549 cells via inhibition of mPGES-1 (IC $5_{50}=0.49 \mu \mathrm{M}$ in a cell-free assay) together with high selectivity over both COX-1 and COX-2. A molecular docking study theoretically suggests that MPO-0186 could inhibit PGE2 production by blocking the $\mathrm{PGH}_{2}$ binding site of the mPGES-1 enzyme. Furthermore, MPO-0186 demonstrated good metabolic stability in human liver microsomes and no significant inhibition observed in clinically relevant CYP isoforms.
\end{abstract}

Keywords: mPGES-1; inflammation; arylsulfonamides

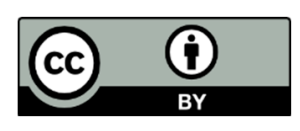

(C) 2019 by the authors. Licensee MDPI, Basel, Switzerland. This article is an open access article distributed under the terms and conditions of the Creative Commons Attribution (CC BY) license (http://creativecommons.org/licenses/by/4.0/). 